\title{
A particular difficulty in discriminating between mirror images
}

\author{
Jules Davidoff*, Elizabeth K. Warrington \\ Department of Psychology, Goldsmiths College, University of London, Lewisham Way, London SE14 6NW, UK
}

Received 11 December 2000; received in revised form 23 February 2001; accepted 27 February 2001

\begin{abstract}
We investigated the selective impairment of mirror image discrimination in a patient with bilateral parieto-occipital lesions (FIM). We report a difficulty with the discrimination between mirror images more selective than has been previously reported (Turnbull $\mathrm{OH}, \mathrm{McCarthy}$ RA. Failure to discriminate between mirror-image objects: a case of viewpoint-independent object recognition? Neurocase 1996;2:63). FIM was asked to judge, in five same/different experiments, whether pairs of simultaneously presented line drawings of objects were identical. FIM demonstrated only a minor impairment in discriminating between orientations in the picture plane but was at chance in making discrimination between mirror images. An experiment with normal observers established that our results were not due to differences in task difficulty. Two further experiments investigated the effects of rotation on the discrimination of letters and geometric shapes. FIM's impairment extended to geometric shapes but not to letters. These results would be consistent with the preservation of an abstract representation for object recognition that did not code the difference between mirror image views. (c) 2001 Elsevier Science Ltd. All rights reserved.
\end{abstract}

Keywords: Object orientation; Object recognition

\section{Introduction}

It has long been known that patients with parietal lesions, and in particular right-sided lesions, demonstrate problems with tasks requiring spatial analysis [34,61]. These difficulties include dealing with object changes both in the picture plane $[13,24,47,54,55]$ and in the depth plane $[38,55,63]$. However, right parietal brain-damage does not invariably lead to impairments in dealing with both types of orientation change [12,54,55]. For example, Farah and Hammond [12] reported that a deficit in mental rotation skills did not prevent accurate object identification even when objects were inverted. Furthermore, and most critical for our study, Turnbull et al. [54] reported patients who were selectively impaired either on inversion or mirror image judgements. Evidence from normal observers also supports the dissociation between the types of orientation change [30].

The case RJ of Turnbull and McCarthy [55] is of particular interest. He was selectively impaired in dis-

\footnotetext{
* Corresponding author. Tel.: + 44-207-9197888; fax: + 44-2079197873.

E-mail address: j.davidoff@gold.ac.uk (J. Davidoff).
}

criminating between mirror images in tasks that might appear to be trivially easy. The patient was not required to perform the mentally difficult task of rotating two objects to determine whether they have different handedness (i.e., were mirror images). RJ was simultaneously presented with three line drawings and merely asked which one was different. Whereas he could do this task if the odd-one-out was inverted, he could not if it was a mirror image. Mirror image discrimination was made even easier for the case (RK) reported in Davidoff and Warrington [9]. In that study, the patient was simply presented with two line drawings and asked if they were the same or different. While it may be easy to understand that patients with spatial problems would not be able to use mental rotation, one might have considered that their ability to make shape or orientation discriminations would be sufficient to judge simultaneously presented mirror images simply as same or different.

The difficulty that RJ and RK showed bears comparison to the common problem children have in learning to discriminate between lateral mirror image pairs of letters [8,10]. Indeed, Rudel and Teuber [42] reported that 3 -year-old children were virtually incapable of 
learning such discriminations. The difficulty is overcome in most people to the point that the task becomes trivial; however, recent data suggest that the primate brain is, in fact, neuronally unprepared to make these discriminations. Neurones in the macaque inferotemporal cortex were found to be invariant to the handedness of the stimulus array [41]. Nevertheless, despite their insensitivity to lateral mirror image pairs (e.g., b vs. d), these neurones were sensitive to vertical mirror image pairs (e.g., b vs. p). Humans, too, find left-right mirror image discriminations considerably more difficult than vertical mirror image discriminations $[8,40]$. The present paper extends these findings by considering the mechanisms subserving object recognition in a patient for whom the ability to make mirror image discriminations is impaired.

Two types of representations that have been proposed for object recognition. The types of representation in which different object views, including the mirror image, are coded separately are termed view-specific $[33,50]$. Models of object recognition based on such representations are contrasted with those that require, or emphasise, representations to be view-independent $[3,33]$. Biederman's model, for example, is based on basic object parts (geons) for which mirror images may be treated as equivalent in recognising an object. These representations are similar to the canonical view representations investigated in our previous research $[9,63]$. When the task entails naming, data from normal observers imply that we use only the canonical view representations. Hence, the facilitation in naming latency from priming is invariant with respect to mirror images $[4,28,29]$ though this may be modulated by attention [49].

The neuropsychological evidence is compatible with the data from normal observers because an inability to make mirror image discriminations does not prevent good object identification of canonical view objects [9,53-55]. In the present paper, we consider whether the difficulty of mirror image discriminations could derive from their rather limited importance for object identification. If an observer were conducting mirror image discriminations solely from canonical view representations there would be no difficulty at all in understanding why mirror image discriminations were so hard. Indeed, a recent report concluded that it was just this inability to disengage from object-centred representations that prevented mirror image discriminations [58].

Our present investigation concerns a patient (FIM) with pronounced difficulty with spatial tasks. The patient bears a striking similarity to the case RJ presented by Turnbull and McCarthy [55]. RJ showed impaired mirror image discrimination with the preservation of orientation for inversion judgements but that was the only misorientation contrasted with mirror images. Our investigation will compare mirror image discriminations to changes in orientation in the picture plane. Recent evidence suggests that not all orientations are equally represented in object representations. For example, in monkey, Ashbridge et al. [1] showed that a greater number of head and body cells were coded for the upright. Thus, recognition of non-upright views of heads and body stimuli could take longer because cell activity to these orientations would be weaker. Karnath et al. [24] thereby argued that upright views of other types of visual stimuli might be preferentially coded and, for that reason, make inversion judgements less sensitive to neuronal insult. We will, therefore, widen the number of orientation changes to be compared with mirror image discrimination. Our first aim was, therefore, to consider the selective impairment for mirror image discriminations. It would be even more remarkable, if the impairment were present when the more difficult picture plane orientation discriminations were found to be intact.

Our second aim was to address questions of object representation in three ways. First, in many object views, determining the orientation of the principal axis would be sufficient to differentiate an object from its mirror image (Fig. 1). We investigated those displays to ask whether a mirror image impairment was present even when the orientation judgement was within the capacity of the patient. Thus, we examined whether an impairment in mirror image discrimination was present when the spatial demands of the task should predict otherwise. Second, we considered mirror image discriminations of non-objects. Most of the previously used stimuli involve object representations but, in our paradigm, successful performance could be achieved from mechanisms involving shape discrimination or spatial frame analysis. Therefore, we compared mirror image tasks using familiar objects to performance with geometrical figures. Third, we considered particular

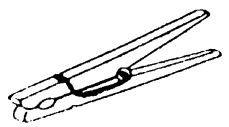

a)

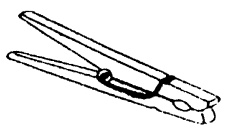

b)

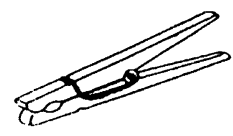

c)

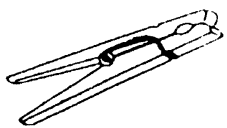

d)
Fig. 1. Example of non-base object discriminations: (a) and (b), mirror image; (c) and (d), inversion. 
types of display (e.g., letters) for which handedness must be coded for correct identification.

\section{Case report}

FIM, a 35 year old Bangledeshi housewife, (resident in England for 10 years) was admitted to a general hospital 9 days post partum in December 1992. She had a severe frontal headache and was drowsy and disorientated. Over the next 10 days her condition deteriorated and she developed a left-sided weakness and a rightsided hemiparesis. At this time, she was transferred to the National Hospital, Queen Square. A MRI scan demonstrated bilateral infarctions of the parietal-occipital regions extending to the left frontal lobe; this was considered to be due to a cerebral venus sinus thrombosis. She made a slow recovery and, on examination at the time of her discharge to the rehabilitation unit in February 1993, she had a wide-based gait, a minor residual right hemiplegia, weakness of the left leg, a homonymous left inferior quadrantinopia and a degree of visual disorientation that impaired her point localisation and depth perception (for further details see [26]).

At the time of the present investigation, begun in July 1998, she had continued to improve and had achieved a degree of independence in the activities of daily life. The inferior left quadrantinopia was still present.

\section{Neuropsychological assessment}

English was her second language, which she had learned to a reasonable level. Her vocabulary was fairly extensive. Neither in naming tasks nor in propositional speech was any paraphasic responses noted. She was not left-right disoriented on her own body. Our assessment of her cognitive abilities focused on her visualspatial and perceptual abilities.

\subsection{Early visual processing}

Visual acuity was measured at $6 / 6$ on the Ffoukes symbols test. On the Efron shape test, she scored 20/20 on an easy discrimination (square $2^{\prime \prime} \times 2^{\prime \prime}$ versus oblong $\left.1.5^{\prime \prime} \times 2.5^{\prime \prime}\right)$ and $17 / 20$ on a more difficult discrimination (square $2^{\prime \prime} \times 2^{\prime \prime}$ versus oblong $1.8^{\prime \prime} \times 2.2^{\prime \prime}$ ); this is marginally weak. On a subsequent occasion, she scored $8 / 8$ for the more difficult discrimination. She was also presented with a series of five ovals all having a ratio of axes 1.7:1. These ovals reduced in size from the largest, having a principal axis of $6 \mathrm{~cm}$, in steps of approximately $0.5 \mathrm{~cm}$. She was able to rank/order by size the series of five ovals. In a further test, her ability to discriminate the larger of two adjacent ovals was satisfactory $(5 / 5$ correct).
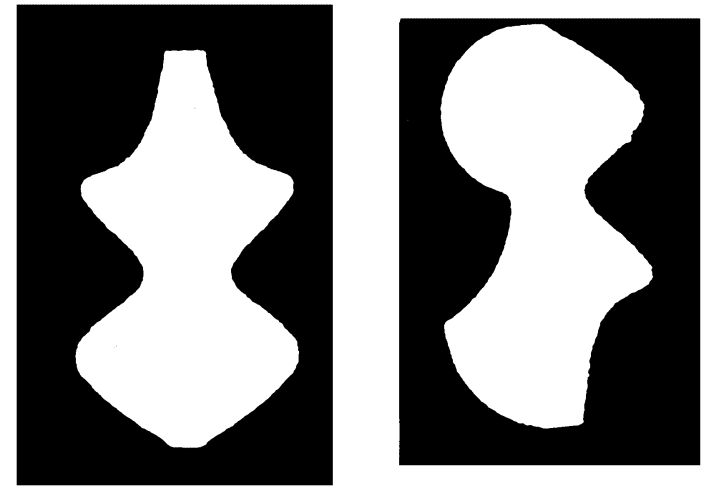

Fig. 2. Example of symmetrical and asymmetrical shapes.

Her ability to judge whether figures were symmetrical was assessed by showing white geometric shapes on a black background. Five of these figures were symmetrical and five (made from different halves of the same symmetrical figures) were asymmetrical (Fig. 2). The figures were also presented upside down to give a total of 20 presentations. FIM correctly identified the figures as symmetric or asymmetric 19/20 times.

FIM obtained a perfect score on the shape detection test of the visual object and space perception (VOSP) $(20 / 20$ correct). She was able to detect the illusory contours of the Kanizsa figures and was able to name correctly eight basic colours [2].

\subsection{Object recognition}

FIM attempted the object perception tests of the VOSP [61] (Table 1). In contrast to her fairly satisfactory performance on the tests of early visual processing, she scored at an impaired level on all four tests of object recognition. She also attempted a version of the Unconventional Views Object Recognition Test on which she scored $7 / 20$, which represents a marked impairment. By contrast, she was able to identify and name $19 / 20$ corresponding conventional views, which is a normal score. Her satisfactory ability to identify and

Table 1

Perceptual and spatial test scores ${ }^{\mathrm{a}}$

\begin{tabular}{lrc}
\hline & Score & $5 \%$ cut off \\
\hline Silhouettes & $14 / 30$ & $<16$ \\
Progressive silhouettes & $14 / 20$ & $>14$ \\
Object decision & $9 / 20$ & $<15$ \\
Fragmented letters & $17 / 20$ & $<17$ \\
Position discrimination & $8 / 20$ & $<18$ \\
Dot counting & $3 / 10$ & $<8$ \\
Number location & $0 / 10$ & $<7$ \\
Cube analysis & $2 / 10$ & $<6$
\end{tabular}

a FIM's test scores on the visual object and space perception (VOSP) battery with $5 \%$ cut-off scores for standardisation sample. 
name canonical object representations was further demonstrated by her ability to name the majority of the Snodgrass and Vanderwart [46] corpus of line drawings (212/260 correct). The majority of her errors were misperceptions. Thus, FIM clearly demonstrates a discontinuity between her very poor performance on tasks in which the perceptual difficulty has been manipulated and her competent performance in identifying canonical object representations; this is the hallmark of an apperceptive agnosia.

She was also tested on the recognition of object parts ([9] Exp. 1). In this study, observers are required to identify, from an object part, the object from which the part is taken. The parts are graded as hard, middle and easy difficulty for identification. The examination followed the procedure of the earlier study. Of the 72 items for which data are presented in Davidoff and Warrington [9], FIM recognised 12 hard (16.7\%), 29 middle $(40.3 \%)$, and 44 easy $(61.1 \%)$ items. The three parts were also presented together in an 'exploded form' [9]; 51 items $(71.8 \%)$ were recognised in this condition. However, a prediction of correct recognition based on her probability of being able to recognise any of the parts would predict a recognition score for FIM of 55 items $(76.4 \%)$. It could well be that her inability to integrate items across space would prevent her benefiting from the simultaneous presentation of all three parts. FIM recognised $71(98.6 \%)$ of the items presented as whole objects. Though FIM performed marginally better than the patient RK in object part recognition [9], she clearly is markedly impaired at the task.

FIM's ability to tell the whether an object was upright was largely unimpaired. Twelve base objects were each presented at a random orientation and FIM was asked to rotate them to their correct position; this was then repeated. She was generally accurate (within $10^{\circ}$ ) only making one error that was more than $45^{\circ}$ incorrect. Thus, the patient showed a quite different pattern of performance to those that have been termed orientation agnosic $[48,54]$.

\subsection{Space perception}

Her point localisation was assessed on the Aimark perimeter. Within $10^{\circ}$ of the fovea she made only minor displacement errors. FIM's performance on the four spatial subtests of the VOSP was seriously impaired (Table 1). She attempted an adaptation of the Flags Test of Space Thinking. One pattern, which has been rotated, has to be matched as identical to one of two choices (Fig. 3). She appeared to be totally unable to comprehend this task scoring only $4 / 10$ before it was discontinued. On the Corsi Span task [64] she was able to attempt strings of three items reasonably accurately but she failed with strings of four blocks. She was able
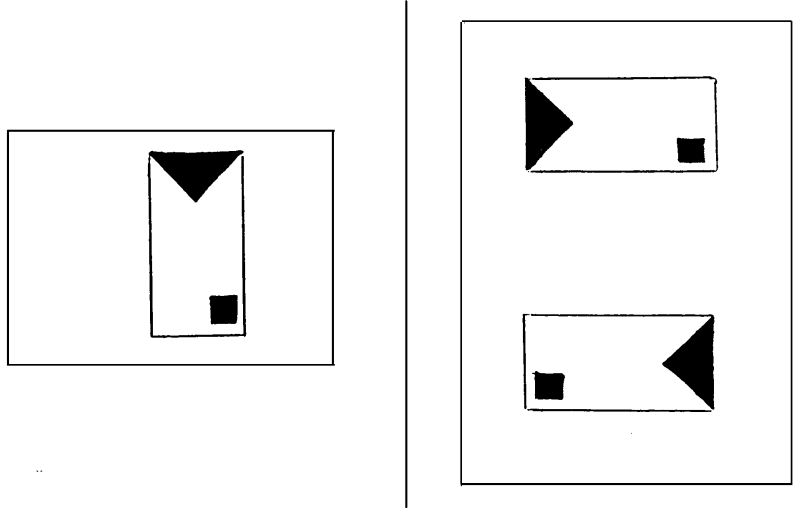

Fig. 3. Example of 'Flags' test of mental rotation.

to copy/tap single taps reasonably accurately (8/9 correct).

An adaptation of Benton's line orientation task was devised; two sloping $6.4 \mathrm{~cm}$ lines were arranged within a $14.8 \mathrm{~cm}$ square such that 20 stimuli were parallel, 20 differed by $5^{\circ}$ and 20 by $10^{\circ}$. She scored $12 / 20,15 / 20$ and $19 / 20$, respectively on a same/different judgement. In summary, FIM's visual disorientation has largely resolved; however, she still has major visual-spatial impairment characteristic of a visual-spatial agnosia.

\section{Experiment 1: discrimination of object orientation in normal observers}

\subsection{Introduction}

We intend using a same/different procedure with FIM to investigate mirror image discrimination. In order to rule out concerns about difficulty level, our preliminary experiment investigates displays to determine the relative difficulty of mirror image to picture plane rotation discriminations. Without such control of the stimuli there remains the possibility that any difficulty for mirror image discriminations arises from a magnification of the small differences that normal observers may have with this particular discrimination compared to, say, inversion discriminations. We, therefore, tested normal observers in a task identical to those to be given to FIM.

Our experimental procedures will be strengthened by the use of the non-base objects (e.g., scissors, spanner) introduced in Davidoff and Warrington [9] to investigate the effects of inversion. A recent study has further justified making the distinction between base and nonbase objects [57]. It was found, for base objects that increasing the rotation in the picture plane systematically impaired an object/non-object decision; this was not the case for non-base objects [57]. We now promote the use of non-base objects for the examination of 
mirror image discriminations. It appeared to us that, when inverted, these non-base objects provide stimuli that are harder to discriminate from the original than a mirror image transformation (Fig. 1). So, using these stimuli would add weight to any claim for a selective impairment for mirror images not being due to the task being more difficult.

\subsection{Observers}

Twenty observers (nine male, 11 female) with an age range of 24-39 years completed the experiment. All were members of staff recruited from hospitals in the London area.

\subsection{Stimuli}

Line drawings of 24 objects with a reliable base (base objects) and 24 objects (Appendix 1 and Fig. 4) seen frequently at many orientations (non-base objects) were obtained from the Snodgrass and Vanderwart [46] set. They were scanned for use in a lap-top computer. Stimuli were presented in pairs. A pair consisted of one of the objects and the same object either presented in an identical orientation or at a different orientation.

\subsection{Procedure}

Observers were given the following instructions: 'In this experiment, you will be presented with pairs of images. Your task is to determine if the images are the same or different. Roughly, half are the same and roughly, half different. When the pairs are different, one of the images will be at a different orientation or perhaps as a mirror image. These differences will be easy to see. You are not looking for subtle differences. If the images are the same, then you should press the 'same' key and if different, the 'different' key. You should respond as quickly and as accurately as possible'.

The same pairs were presented at $0^{\circ}, 45^{\circ}, 90^{\circ}$, inverted, as from their orientation in Snodgrass and Vanderwart [46]. It should be noted that for non-base items this ranged from approximately $30-45^{\circ}$ from the horizontal. Each pair was presented side by side on each side of the midline and covered roughly $5 \times 5 \mathrm{~cm}^{2}$ on the screen. They were observed from around $30 \mathrm{~cm}$. There were 48 same pairs at every orientation with equal numbers of base and non-base items except that in order to present equal numbers of mirror image stimuli the number of same stimuli presented at $0^{\circ}$ was 96. For different stimuli, the orientation change from $0^{\circ}$ was equally often $45^{\circ}, 90^{\circ}$, inverted or as a mirror image. The $0^{\circ}$ version for each pair was half the time on the right of the screen and half the time on the left.

Each observer saw all 432 pairs in a different random order. For half the observers, the 'same' key was on the left and for half it was on the right. The images remained on the screen until observers gave their response.

\subsection{Results}

Latencies and accuracy scores, after removing incorrect responses and those more than two standard deviations from the mean of each condition are shown in Fig. 5(A) and (B).

Latencies for different responses were analysed in a 2 (Base: Base vs. Non-base) $\times 4$ (Orientation: 45 vs. $90^{\circ}$ vs. inverted vs. mirror) analysis of variance with repeated measures over both factors. There was an effect of orientation $(F(3,57)=44.00, P<0.001)$ and an interaction $(F(3,57)=10.01, P<0.001)$. An analysis of the interaction (Fig. 5(A)) showed that, for base objects, mirror image discriminations were harder than inverted discriminations $(t(19)=4.27, P<0.001)$ though this was not the case for non-base objects $(t(19)=1.86$, $P>0.05)$; indeed, Fig. 5(A) shows that the latencies are in the opposite direction. The 45 and $90^{\circ}$ discriminations were performed faster than the other two orientation changes for both base and non-base objects (all $P<0.05)$. Inspection of Fig. 5(B) shows that the error data are in the same direction as the significant effects found in the analysis of latencies. However, errors were rather few and even after an arcsin transformation the analysis of variance gave no reliable effects (all $P>$ 0.2 ).

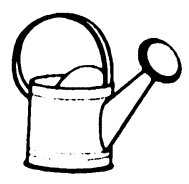

a)

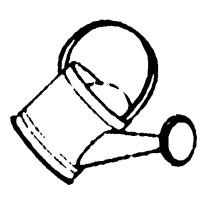

b)

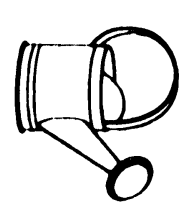

c)

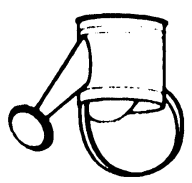

d)

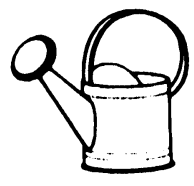

c)

Fig. 4. Example of base object in 5 orientations used in Experiment 1: (a) $0^{\circ}$; (b) $45^{\circ}$; (c) $90^{\circ}$; (d) inverted; (e) mirror. 

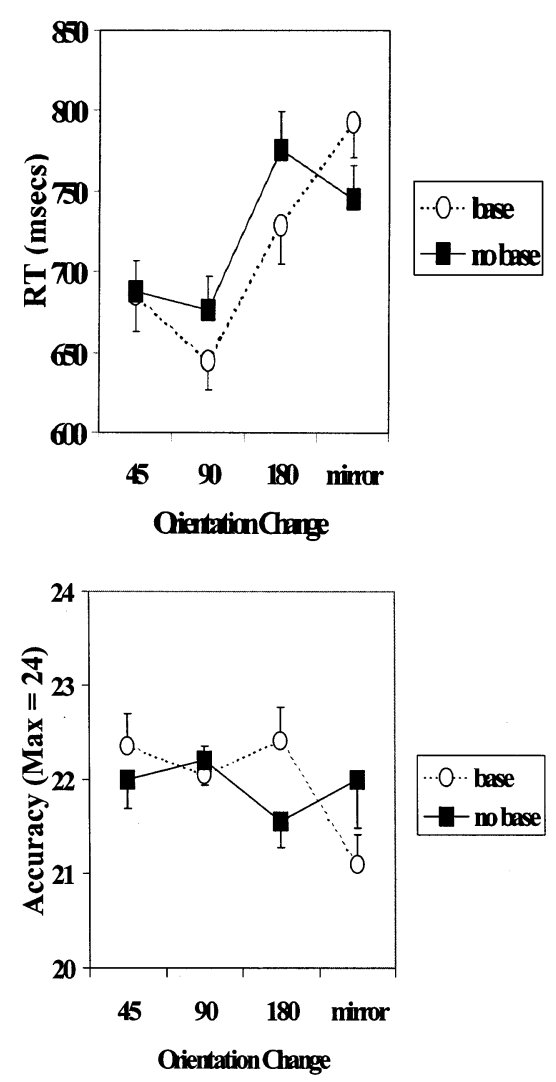

Fig. 5. (A) Latencies and (B) accuracy scores for normal observers in the discrimination of orientation differences between object pairs.

\subsection{Comment}

Experiment 1 established that, for normal observers, mirror image discriminations are not always more difficult than inversion discriminations. In particular, for non-base objects, inversion judgements are harder than mirror image discriminations. Therefore, we can be reassured that, when these studies are carried out on FIM, any significant dissociations between mirror images and picture plane orientation discrimination cannot be merely attributed to difficulty level.

\section{Experiment 2: orientation discrimination in FIM: comparison of mirror image to inversion}

\subsection{Introduction}

Our aim in this experiment was to document FIM's ability to detect mirror image rotations and plane rotations of meaningful object stimuli.

\subsection{Stimuli and procedure}

The stimuli were the same 48 pictures of asymmetric non-animate items from the Snodgrass and Vanderwart [46] set used in Experiment 1. Following the experimen- tal design used previously, FIM was asked to respond same or different to pairs of stimuli. There were two conditions of stimulus presentation: 'blocked' and 'random'. For the blocked condition, the stimuli were arranged in four sets of 24 items as follows: (a) 12 base objects paired with its mirror rotation and 12 identical pairs, (b) 12 base items paired with its inverted rotation and 12 identical pairs, (c) 12 non-base items paired with its mirror rotation and 12 identical pairs (d) 12 non-base items paired with its inverted rotation and 12 identical pairs. For the 'random' condition, 16 of the base objects and 16 of the non-base objects were paired with a mirror image rotation, 16 of base and 16 of the non-base objects were paired with the inverted rotation and the remaining 16 of each type of object pair were identical. These 96 stimuli were arranged in random order and, in order to prevent fatigue, presented in three sets of 32 items. It should be noted that in this presentation condition only one third of the pairs were identical whereas, in the blocked condition one half of the pairs were identical.

\subsection{Results}

The percent correct for the 'blocked' condition for each type of paired comparison $(n=24)$ was as follows: mirror rotation base object, $48 \%$; mirror rotation nonbase objects, $42 \%$; inverted rotation base objects, $96 \%$; inverted rotation non-base objects, $85 \%$. There is a highly significant difference between her inability to judge mirror rotations as compared with relative intact performance with inversion rotations; this difference was very similar for both base and non-base stimuli.

The percent correct for each type of paired comparison presented in the 'random' condition is given in Table 2. Again FIM is very reliable in judging inversion rotation pairs to be different but has significant difficulty in judging mirror image rotations to be different. However, this is unlikely to reflect a response bias towards making 'same' judgements to all but the inversion rotation pairs since she judged over half of the pairs that were the 'same' to be different.

Table 2

Discrimination of mirror and inversion rotations ${ }^{\mathrm{a}}$

\begin{tabular}{llll}
\hline & $\begin{array}{l}\text { Mirror } \\
\text { rotation (\%) }\end{array}$ & $\begin{array}{l}\text { Inverted } \\
\text { rotation (\%) }\end{array}$ & Identical (\%) \\
\cline { 2 - 4 } & \multicolumn{3}{c}{$[3] n=16$} \\
\cline { 2 - 4 } $\begin{array}{l}\text { Base objects } \\
\begin{array}{l}\text { Non-base } \\
\text { objects }\end{array}\end{array}$ & 31 & 94 & 44 \\
\hline
\end{tabular}

a FIM's scores (\% correct) for base and non-base objects in the mirror and inversion rotation conditions tested in randomised order (Experiment 2). 


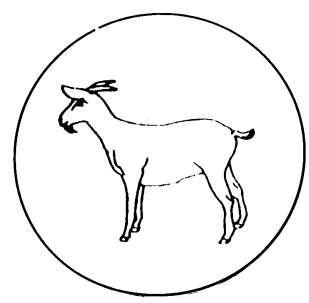

a)

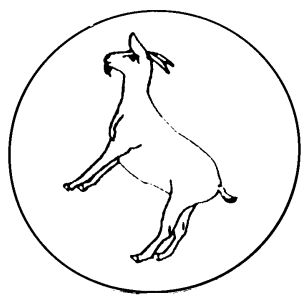

b)

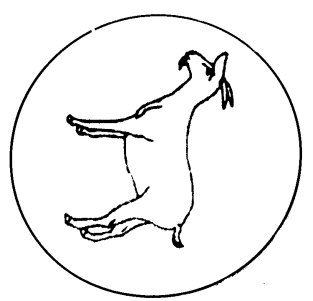

c)

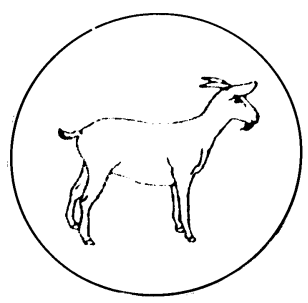

d)

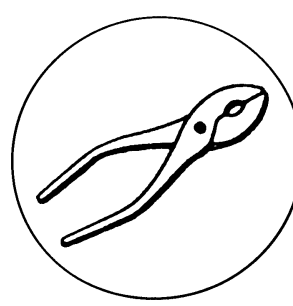

e)

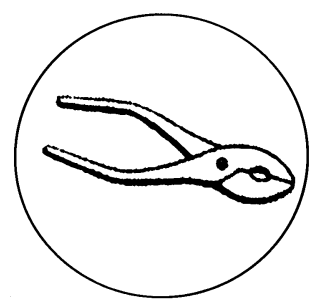

f)

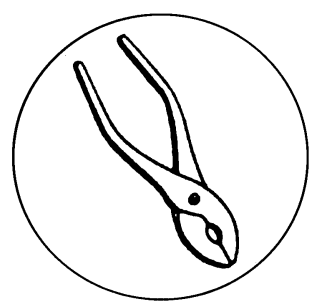

g)

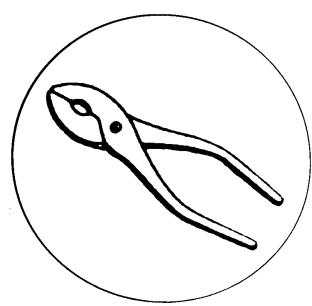

h)

Fig. 6. Examples of base and non-base objects presented on circular cards: (a) and (e) $0^{\circ}$; (b) and (f) $45^{\circ}$; (c) and (g) $90^{\circ}$; (d) and (h) mirror as used in Experiment 3.

\subsection{Comment}

We have documented a very significant inability to differentiate an object from its mirror image. This difficulty cannot be attributed to the spatial demands of the task that require her to compare an array of two stimuli. Similar arrays were used for the inversion rotation conditions and this presented her with very little difficulty. Indeed, her ability to distinguish between the non-base items in the inversion rotation but not with the mirror rotation was particularly striking since with these stimuli there are not the added cues of normal verticality to provide extra information.

\section{Experiment 3: orientation discrimination in FIM: comparison of mirror image to 45 and $90^{\circ}$}

\subsection{Introduction}

Our aim in this experiment was extend our findings of Experiment 2 by comparing FIM's ability to distinguish plane rotations other than inversions with her ability to distinguish mirror image rotations.

\subsection{Stimuli and procedure}

The test stimuli consisted of 48 pictures; 24 animals, 12 mostly new objects with a base and 12 mostly new objects without a base, selected from the Snodgrass and Vanderwart [46] set (Appendix 1). The 45 and $90^{\circ}$ rotations were compared with mirror image rotations
(Fig. 6). Every stimulus item was presented in each rotation condition and with an identical stimulus. The stimuli were prepared on circular white cards and presented on a much larger white background card. The background card contained discrete alignment marks to ensure accurate orientation of stimuli.

Time constraints did not make it feasible to adopt a fully randomised testing procedure; consequently, this experiment was completed in two stages. In stage 1 , pairs of stimuli having either a $90^{\circ}$ rotation, a mirror image rotation or being identical were presented in a randomised order making a total of 144 stimulus pairs. In stage 2, pairs of stimuli were presented having either a $45^{\circ}$ rotation or being identical making a total of 96 stimulus pairs. Thus, in stage 1 , a third of the stimuli were identical pairs and in stage 2 half were identical pairs. As before, FIM was asked to say whether the stimulus pairs were identical or different.

\subsection{Results}

In Stage 1, the percent correct for each type of stimulus pair $(N=48)$ was as follows: $90^{\circ}, 100 \%$; mirror rotation, 58\%; same $92 \%$. In Stage 2, the comparison between $45^{\circ}$ and same pairs $(N=48)$ gave $45^{\circ}$, $83 \%$; same $79 \%$.

FIM's performance is again most impaired for the mirror image condition. Her performance on the $90^{\circ}$ rotation condition was at ceiling and with the $45^{\circ}$ rotation fairly satisfactory and significantly better than with the mirror rotations. The apparent bias to giving 'same' responses may have been in part influenced by 
her obvious ability to detect differences with the plane rotations.

We note that FIM has a minor difficulty in telling whether base objects are upright and this may be contributing to her errors at $45^{\circ}$. A more fine grain analysis of her errors in the $45^{\circ}$ rotation condition brought to light an otherwise unexpected finding. She had significantly more difficulty in detecting rotation with the base stimuli, all of which had a strong horizontal axis. It was also notable that she scored $23 / 24$ on the non-base items despite the fact that none of these stimuli had firm horizontal or vertical axes which might be assumed to contribute to the accuracy of such discriminations.

\subsection{Comment}

We have established that FIM's ability to detect plane rotations of objects is relatively preserved and contrasted with mirror image rotations is strikingly superior. We were particularly impressed by her performance with the non-base items. Despite having no additional cues of verticality or horizontality even here she detected $45^{\circ}$ plane rotations more accurately than the mirror image rotations. Yet to the experimenters, for the non-base items, the differences between the mirror rotations were often more obvious than with the plane rotations (Fig. 6).

\section{Experiment 4: non-base items comparison of mirror image to $90^{\circ}$ and inversion}

\subsection{Introduction}

In Experiment 3, there was only a relatively small number of non-base items. Our aim in this experiment was to compare plane rotations with mirror rotations using a larger set of non-base objects not having a conventional or usual vertical orientation. For such objects, a $90^{\circ}$ or even an inversion is as familiar as what was deemed to be the upright orientation.

\subsection{Stimuli and procedure}

The stimuli consisted of 24 non-base asymmetrical items selected from the [46] set. Twelve of these stimuli were drawn from those used in Experiment 1 and 12 from those used in Experiment 3. Each item was paired with its mirror image rotation, a $90^{\circ}$ rotation, an inversion and with an identical stimulus. These 96 stimulus pairs were presented in random order in a single session and FIM was required to make a same or different response. Otherwise, the procedure was as in Experiment 3.

\subsection{Results}

Her performance was flawless for the $90^{\circ}$ rotation $(24 / 24$ correct), for the inverted rotation $(24 / 24$ correct) and for the identical pairs (24/24 correct). All her errors occurred on the mirror rotation condition (16/24 correct).

\subsection{Comment}

Many of the stimuli in the upright orientation were presented with the major axis at a diagonal. The mirror image rotation of such stimuli changed the spatial co-ordinates of the display, whereas the inverted rotation did not. Despite this additional spatial cue for detecting the mirror image rotation it was for these stimulus pairs and only these stimulus pairs that errors occurred. We would suggest that this finding is very powerful evidence of the special nature of mirror image representations.

\section{Experiment 5: FIM's discrimination of vertical mirror images}

\subsection{Introduction}

Experiments 2-4 have shown that FIM has particular difficulty with mirror image discriminations but the relative preservation of orientation discrimination in the picture plane. In considering the primate's difficulty in making mirror image discrimination, Rollenhagen and Olson [41] distinguish between lateral mirror image discriminations (those used in Experiments 1-4) and vertical mirror images. Monkeys had considerable difficulty with lateral mirror image discriminations but found vertical mirror image discriminations easy. Therefore, we examined FIM's performance on vertical mirror image discriminations.

\subsection{Stimuli}

Twenty-four base and non-base stimuli (a subset of the stimuli used in Experiment 1) were randomly presented with either identical stimuli or with its vertical mirror image (Fig. 7) giving a total of 48 presentations.

\subsection{Comment}

FIM made no errors. The poor performance of FIM with lateral mirror image discriminations does not extend to distinguishing an object from its vertical mirror image. Thus, the pattern of performance is identical to that found in Rollenhagen and Olson [41]. 


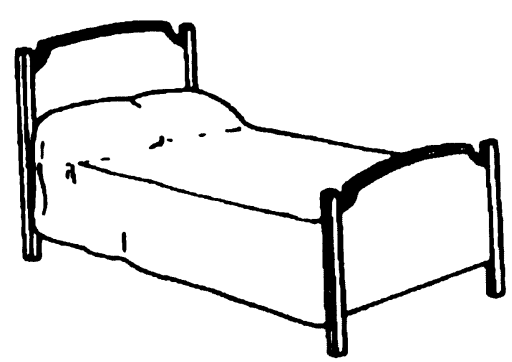

a)

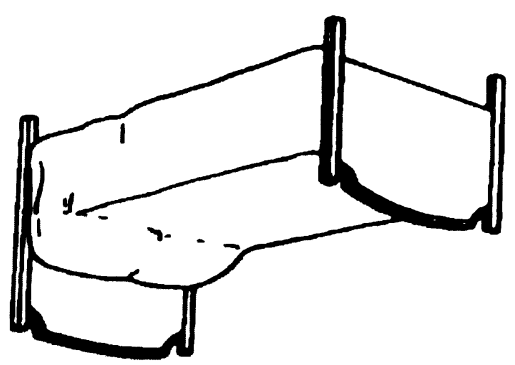

b)

Fig. 7. Example of discrimination of object and vertical mirror image as used in Experiment 5.

\section{Experiment 6: FIM's orientation discrimination with symmetrical objects}

\subsection{Introduction}

Our neuropsychological assessment of FIM showed that her ability to detect differences in line orientation was likely to be satisfactory for differences greater than $10^{\circ}$. FIM was also able to discriminate, in Experiment 3 , between many objects that differed by $45^{\circ}$ in the picture plane. It could well be argued that her relatively preserved orientation discrimination should promote better performance with the mirror image non-base displays than revealed in the previous experiments. We, therefore, devised stimuli that might encourage matching by orientation. In previous experiments, items were chosen that were clearly asymmetrical about both principal axes; in this experiment, we used stimuli that were symmetrical about one axis.

\subsection{Stimuli and procedure}

For this experiment, we used objects symmetrical about one axis for which a mirror image can also be produced by a plane rotation. We, thus, made available to FIM another procedure by which she could effect a same/different judgement of mirror images.

We first confirmed that FIM would clearly differentiate between lines at the orientations investigated in Experiment 2. Pairs of lines were presented that were either at the same orientation $\left(45^{\circ}\right)$, differed by $45^{\circ}$ (i.e. one was vertical) or one was a mirror image (i.e. differed by $90^{\circ}$ ). Ten of each of these pairs were randomly presented in a same/different task. FIM made no errors.

FIM was then presented with 12 items symmetrical about the vertical but not the horizontal axis from the Snodgrass and Vanderwart set (Appendix 1). In most of the cases, the symmetry was not perfect until the stimuli were adapted in PнотоSнор. These stimuli give dis- plays that when presented as mirror images are identical to a rotation in one direction in the picture plane. Stimuli were presented on circular cards as in Experiment 3. Three conditions were produced in which an item presented with its principal axis at $45^{\circ}$ was compared to an identical item, its mirror image and to an 'upside down' condition in which the item was rotated so that its principal axis was at the same orientation as the mirror image (Fig. 8). The conditions were first blocked with 'same' being compared to mirror images. The three conditions were then presented at random.

\subsection{Results}

For the blocked trials, FIM made $7 / 24$ errors, when discriminating between 'same' and mirror image trials. Discriminating between 'same' and upside down trials; she made only $1 / 24$ errors $\left(\chi^{2}=5.4, P<0.05\right)$. In the randomised trials, FIM made no errors on upside down discriminations, $3 / 12$ errors on same discriminations and was at chance with mirror image displays (6/12 errors).

\subsection{Comment}

Despite the fact that her orientation ability should have provided FIM with sufficient information to perform the task, when lines were replaced with symmetri-

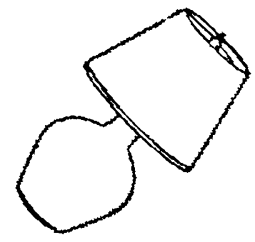

a)

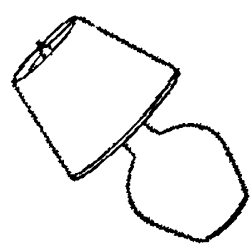

b)

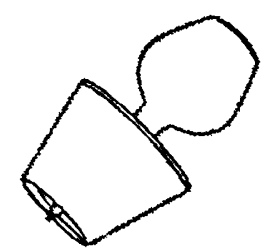

c)
Fig. 8. Example of symmetrical object: (a) $0^{\circ}$; (b) mirror image; (c) inverted as used in Experiment 6. 


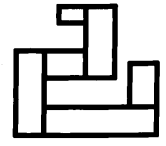

a)

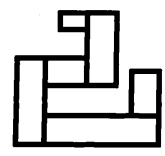

c)

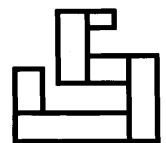

b)

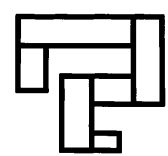

d)
Fig. 9. Example of geometrical shape discriminations: (a) and (b) mirror; (c) and (d) inversion as used in Experiment 7.

cal objects, she once again began to fail with mirror image discriminations. The mirror image presentations appear to override her preserved orientation skills.

\section{Experiment 7: FIM's orientation discrimination with geometric figures}

\subsection{Introduction}

In Experiment 6, we recorded further evidence of FIM's selective difficulty in detecting mirror image rotations. Our aim in this experiment was to establish whether there would be the same pattern of performance with meaningless geometrical figures.

\subsection{Stimuli and procedure}

The test stimuli consisted of 15 complex meaningless geometrical figures (Fig. 9). Each figure was paired with its mirror image rotation and with inverted rotation image. Each of these conditions was tested in 'blocks' with an equal number of identical pairs. There were thus a total of 60 trials and these two 'blocks' were represented on a second occasion.

\subsection{Results}

The percent correct for each condition for each trial is given in Table 3. On trial 1, there was a strong trend for a higher error rate for the mirror rotations than the inverted rotation $\left(\chi^{2}=3.35, P<0.1\right)$ and on trial 2 , the difference between the two conditions was significant $\left(\chi^{2}=7.7, P<0.01\right)$. The difference between the total scores for the two conditions was also significant $\left(\chi^{2}=\right.$ 10.2, $P<0.01)$.

\subsection{Comment}

We have obtained a very similar pattern of results with geometrical figures as with meaningful stimuli. FIM is able to detect inversion rotation significantly more reliably than mirror image rotations. This finding extends the generality of FIM's impaired mirror image discrimination to non-representational stimuli. We will return to this issue in the Section 12.

\section{Experiment 8: FIM's orientation discrimination of alphanumeric stimuli}

\subsection{Introduction}

We have established that FIM's inability to detect mirror images as compared with plane rotations occurs for both meaningful object stimuli and for non-meaningful shapes. It therefore becomes of interest to consider whether this would also apply to well-known verbal symbols for which handedness is important. Many letters and numbers are asymmetric about the vertical axis. The conventional right/left orientation though difficult to acquire becomes an overlearned skill. Our aim in this experiment was to assess FIM's ability to detect mirror image rotations compared with inversion rotations of letter stimuli.

In a pilot study, FIM was presented with 20 pairs of asymmetrical letters $(\mathrm{C}, \mathrm{D}, \mathrm{E}, \mathrm{F}, \mathrm{G}, \mathrm{K}, \mathrm{L}, \mathrm{P}, \mathrm{R}, \mathrm{S})$ in which ten were paired with its mirror image and ten were identical. FIM was asked to respond same or different and her performance was flawless (20/20).

\subsection{Stimuli and procedure}

The test stimuli consisted of the letters F, G, J, L, P, $\mathrm{R}$, and the numbers 4, 5. Each stimulus was paired with a mirror image rotation, an inversion rotation and with the identical stimulus. In addition, each inverted stimulus was paired with its mirror image rotation and an identical stimulus. There were thus a total of 32 stimulus pairs which were presented in a randomised order and FIM was again asked to respond same or different.

Table 3

Discrimination of mirror rotations and inverted rotations for geometrical shapes ${ }^{\mathrm{a}}$

\begin{tabular}{lll}
\hline & Mirror rotation $(\%)$ & Inverted rotation(\%) \\
\hline Trial 1 $(n=30)$ & 67 & 86 \\
Trial 2 $(n=30)$ & 70 & 97 \\
Total $(n=60)$ & 68 & 92
\end{tabular}

${ }^{\text {a }}$ FIM's scores $(\%$ correct) for mirror and inverted rotations (Experiment 7). 


\subsection{Results}

FIM obtained 29/32 (91\%) correct responses. Of the three errors, she judged two inverted Ps to be different and an inverted mirror image pair of a $\mathrm{G}$ and an $\mathrm{L}$ (i.e., paired with the inverted letter) to be the same.

\subsection{Comment}

With these letter stimuli, FIM's ability to detect mirror image rotations improved dramatically and indeed the only errors occurred with the inverted stimuli. FIM demonstrated an entirely satisfactory ability to detect mirror rotations with verbal symbols that have a conventional handedness structure. FIM has no difficulty with letter recognition and is therefore presumably able to reject the mirror images as incorrect letters. This finding reinforces the selective difficulty she has in detecting mirror image rotations of objects that do not have a fixed handedness.

\section{General discussion}

We present here data from a patient who showed a marked problem with mirror image discriminations compared to discriminations between inversions or other plane rotations. Our aims for this study were first to rule out artifacts that might limit the claim for selective impairment and second to identify the functional locus for the disorder. Considering our first aim, Experiment 1 examined the effects of rotation on the base and non-base objects introduced in Davidoff and Warrington [9]. It was shown that normal observers found inversion discriminations harder than mirror image discriminations for non-base objects but not for base objects. However, FIM found mirror image discriminations harder than inversion discriminations for both base and non-base objects (Experiments 2 and 4). These experiments thereby rule out the possibility that FIM's failure with mirror image discriminations was a matter of difficulty level. The selectivity of FIM's impairment was examined in Experiments 3 and 4. In those experiments, it was showed that FIM had no difficulty in making object orientation discriminations over $45^{\circ}$ in the picture plane. Thus, we have shown in FIM a particularly selective impairment for mirror image discriminations.

Our second aim concerned the significance of impaired mirror image discrimination to models of object recognition. FIM's poor performance raises the question of how mirror image discrimination is achieved in our same/different paradigm with respect to the alternative routes for object recognition. Corballis and Beale [8] argued that our paradigm does not truly test mirror image discrimination because correct discrimina- tion can be achieved without an assignment of handedness to each stimulus. However, we would argue that our paradigm makes FIM's failure all the more remarkable. It is even more striking that a failure to make mirror image discriminations is revealed, when success could have been achieved by several procedures. It is worthwhile, therefore, to consider those alternative procedures and their contribution to object recognition.

The first procedure, we shall consider for mirror image discrimination is that involving the mental rotation of one object to attempt a fit to the other. There is an appealingly parsimonious proposal that mental rotation explains all processing for object identification that require plane and depth transformations including mirror image discriminations $[18,20,21,37,51,52]$. However, there are good reasons to doubt the role of mental rotation in dealing with most picture plane or depth rotations. With respect to identification after picture plane rotation, recent research has questioned whether mental rotation is fundamentally important [7,16,31]. Lawson and Jolicoeur [31], for example, showed that the relationship between plane rotation and presentation duration required for object identification was not, in fact, linear; whereas, it was linear for making mirror image judgements in a mental rotation task. Lawson [27] argued, therefore, that mental rotation was not used in recognising objects in plane rotation.

The recognition of plane rotated objects, in fact, emphasises the role of inversion resistant features. Repeated naming of familiar objects reduces plane rotation effects $[20,21,23,31,35]$ but practice does not reduce plane rotation effects in deciding which direction an object would face if it were upright [21]. Practice, according to Jolicoeur [22], encourages a feature based discrimination useful for object identification but is irrelevant for a mirror image discrimination task. With practice, subjects learn to recognise objects using features that are invariant to plane rotation. Turnbull and McCarthy [56] also argued, from neuropsychological evidence, that mental rotation skills were not responsible for object identification of misoriented objects. Their patient showed normal mental rotation skills in the Shepard and Metzler [45] task yet, nevertheless, misidentified drawings of objects if they were rotated in the picture plane.

Similar arguments with respect to mental rotation, to those of Turnbull and McCarthy [56] and Lawson and Jolicoeur [32], were used earlier for the recognition of objects rotated in the depth plane. Warrington and James [59] showed that object recognition did not necessarily become more difficult the greater the depth rotation; it more depended upon whether the rotation hindered the identification of object parts. Indeed, mental rotation would be impossible to employ for recognition of an unidentified object. Without knowing the identity of an object, one would simply not know in 
which direction to rotate the object in depth. Of course, there are circumstances where mental rotation could be useful in making discriminations based on depth rotation. One of those circumstances is our same/different mirror image discrimination paradigm. It is only when knowing the identity of an object or, as in our case, being given the sample to match, that mental rotation could be a useful mechanism for mirror image discrimination. However, FIM's disastrous performance on mental rotation tasks would preclude this first procedure to achieve mirror image discrimination.

The second procedure for achieving mirror image discriminations concerns a spatial comparison of the different view-specific representations formed from the mirror images. Since Marr [32], most models make a distinction between object-centred (view-independent) and view-centred (view-specific) representations. Several later models have even made anatomical distinctions between routes that would deal with these two types of representation. $[25,36,60]$. On most of these models, the routes concerned with view-specific representations have been linked with both parietal structures and spatial processing in what is known as the "dorsal route" $[25,36,54]$. However, the role, if any, of spatial analysis in view-specific representations for object recognition is still uncertain on these models. In Milner and Goodale [36], for example, the bilaterally represented viewer-centred representations are available for motor action even in cases of gross failures of object recognition. The neuropsychological account of Warrington [60] originally proposed a serial route involving right parietal structures for viewer-centred object recognition but was revised in a later report to make the right parietal involvement an optional resource that codes knowledge of object parts [43]. The left hemisphere object recognition system was driven by canonical view representations $[9,43]$.

Irrespective of issues concerned with localisation, there are at least two ways that spatial frames could be analysed to achieve a discrimination between mirror images. The first is a metric system that codes in terms of co-ordinates in a spatial frame; the second is a categorical system that codes space according to equivalence classes (e.g., left/right). Kosslyn et al. [25] proposed that the two systems could be mapped on to hemispheric function rather in the way proposed by Semmes et al. [44]. The metric system of spatial analysis would be subserved by right hemisphere structures [62] and the categorical system by left hemisphere structures (though see Ref. [5] for an alternative view of the locus of the two systems).

Using a categorical coding system for mirror image discrimination within our paradigm would be effortful and particularly difficult for FIM. For example, to code mirror images of a dog in a categorical code, she would have to code head to the right and tail to the left in one display and head to the left and tail to the right in the other. Furthermore, her metric coding would make allocating the position of the head in the frame difficult and her poor object part recognition [9] would not help in isolating the parts.

Turning to consider procedures that would allow metric comparisons, we note FIM's abysmal performance on the spatial tasks of the VOSP (Table 1). It would, for example, be difficult for her to differentiate mirror images by using procedures for comparison of positions between two spatial frames. In considering an earlier patient [9], we favoured impairments to such spatially based systems for explaining all object orientation-matching deficits. However, FIM has retained a considerable ability to compare object orientation and we would, therefore, revise our position for the present case where the matching impairment is very much restricted to comparisons between mirror images. We turn instead to the third procedure available to FIM.

The third procedure for achieving mirror image discriminations concerns object identification. In particular, we want to consider the role of view-independent (canonical view) representations. A recent account explicitly dealing with normal object recognition (Stankiewicz et al. [49]) again proposes two routes. One route for object identification uses structural descriptions and the other route for object identification uses a metric analysis of the representation. Its interest for our present study is that the structural description route is unable to make mirror image discriminations while the metric analysis route is capable of making the distinction. We would like to claim ([14]) that the intact left hemisphere produces a bias towards the use of canonical view representations. If object processing takes this direction, then failure to make mirror image discriminations could be a direct consequence. We first amplify on why the present data point to the use of canonical view representations.

The neuropsychological dissociation between accurate object identification and impaired object orientation in the picture plane poses problems for any stage dependent theory of object recognition [54]. For example, it does not fit with Marr [32] who proposed that recognition was dependent on the prior production of view-dependent representations. However, more damaging than impairments with picture plane rotations, allowing the caveats of Karnath et al. [24], are the present data ([55]) on impaired mirror image discrimination. Despite the arguments of Haywood [17], it is difficult to reconcile intact canonical view object recognition with models that promote view-specific information and, therefore, ought to allow mirror image discrimination. Thus, we would argue that FIM, who has great difficulty recognising objects from unconventional views, is using canonical (view-independent) representations for object identification. The present data 
even rule out the possibility that FIM is using view-specific representations by recourse to the recognition of object parts that are known to be less sensitive to the effects of inversion and mirror image rotation. FIM, and also RK in Davidoff and Warrington [9], showed that this mechanism is likely to be unavailable as she was impaired at the recognition of object parts.

The one type of object for which view-independent representations would allow mirror image discrimination is that where neural systems have been coded for handedness as they are for letters and numbers. Thus, the exception to FIM's inability to discriminate between mirror images was for letters and numbers. A similar exception was found in RJ [55]. Also, RK [9] could differentiate mirror image shoes by labelling them 'left shoe' or 'right shoe'. It is, therefore, reasonable to argue that the only occasions where unilateral left-sided lesions produce impairments for mirror images are when they concern objects coded for handedness.[15,19,39,44]. Further confirmation of the different hemispheric processing involved in left/right discrimination compared to other mirror image tasks comes from recent studies that required identification of either the left or right hand on a pictured body $[65,66]$. They found that, for hand identification, the latency to respond is invariant with respect to the orientation of the body [65]. Moreover, cortical activity activated during the task involves left hemisphere sites [66] whereas mental activity that required imagined rotation was found not only to be linearly sensitive to orientation but also to involve right hemisphere activity $[38,66]$.

Our proposal for the use of canonical representations by FIM prompts consideration of the conditions under which use is also made of the spatially based procedures (see above) that allow an analysis within a spatial frame. The spatial analysis required for differentiating mirror images in our paradigm ought not to be particularly difficult. For example, an earlier patient (JBA) despite rather poor shape discrimination was able to distinguish between mirror images [58]. However, JBA was only able to differentiate mirror images if she could not recognise the object or it was a geometric shape. We argued that JBA, having recognised objects from canonical view representations was unable to disengage from them to attempt a spatial analysis. Hence, the inability to discriminate mirror images was present only for objects that she could recognise. We would argue that FIM also showed difficulty disengaging from canonical view representations. In FIM's case, we would argue that it explains why her object orientation abilities do not allow mirror image discrimination and also why it prevented her making mirror image discriminations of geometrical shapes. We will consider each assertion in turn.

In Experiment 6, we showed that FIM was poor at discriminating between mirror images of laterally sym- metrical objects; this is a task at which she might have been presumed to succeed given her reasonably intact line orientation discrimination. The results of Experiment 6 make clear the particular place mirror image discriminations play in object identification. Once identification is attempted from canonical view representations, no difference can be detected between mirror images. Hence, for mirror image stimuli, the orientation discrimination task is apparently 'solved'. For the patient, there is no need to disengage from these representations to attempt orientation discrimination by other procedures even when they are within the capabilities of FIM. However, other differences in object orientation create a mismatch in the object identification procedures and a disengagement takes place to allow spatially based procedures to operate. It would appear (see also supporting data in Ref. [6]) that relatively small discrepancies (around $45^{\circ}$ ) may be all that is required for the canonical route identification system to note the mismatch. Our data would imply that a mismatch is more easily noted for a change in plane orientation than for a mirror image.

We would propose a similar disengagement account for the failure of FIM to discriminate between mirror images of geometric shapes. FIM's shape discrimination (unlike that of JBA) was good and likewise her canonical object recognition. We might speculate that, in those conditions, the use of the more object-based route [49] takes place even for geometric shapes. In this context, it might be helpful to consider the concept of a geon [3]. Geons are held to be a specific set of geometric shapes (volumes) that constitute the basis of all object recognition. It would be unlikely that JBA would be able to analyse geometric shapes into their geon sections to proceed down an object-based route. Hence, not going down that path, her preserved spatial skills were able to carry out the rather simple task of discriminating mirror images for geometric shapes. Further research with FIM, investigating the geon-like properties of meaningless displays, may provide an answer. However, our suggestion at least proposes an answer to the different patterns of performance with geometric shapes for JBA and FIM.

In conclusion, we have demonstrated a rather specific difficulty with mirror image discriminations in a patient whose abilities to make other orientation judgements are relatively preserved. Her failure at mirror image discrimination emphasises the peculiar place lateral mirror images occupy within the visual system. Lateral mirror images would seem to be a special class of stimuli [41] for which we need a great deal of training to distinguish. The length of the training would come as no surprise if Deregowski, et al. [11] are correct in their belief that the brain automatically produces a lateral mirror image of every presented stimulus. Several alternatives offer themselves in our paradigm for resolving 
mirror images. Of these, mental rotation and alternative forms of spatial analysis would be largely unavailable for FIM. However, her ability to differentiate line orientation and object orientation in the picture plane might have been presumed sufficient for her to differentiate between mirror images. It leads us to speculate that FIM does not use her limited spatial skills because her intact left hemisphere object identification procedures produce a bias towards the use of canonical representations. These are completely inadequate for mirror image discriminations. Furthermore, the inability to make the discrimination prevents her from disengaging from these representations to use her residual spatial skills.

\section{Appendix 1}

Experiment 1: The stimuli used were: Base - bed, bicycle, boot, bus, car, chair, couch, cup, gun, iron, ironing board, jug, kettle, lorry, motor-bicycle, pram, rocking chair, saucepan, shoe, suitcase, telephone, television, toaster, watering-can.

Non-base - axe, broom, brush, chisel, comb, fork, glove, hammer, key, knife, lock, mitten, paintbrush, peg, pliers, plug, saw, scissors, screw, screwdriver, spanner, spectacles, toothbrush, violin.

Experiment 3: Animals - bear, bird, camel, cat, chicken, cow, deer, dog, donkey, duck, elephant, fish, fox, giraffe, goat, gorilla, grasshopper, horse, kangaroo, lion, mouse, ostrich, pig, tiger.

Base - basket, cannon, chest of drawers, church, coat, frying pan, harp, hat, jug, record player, roller skate, sailing boat.

Non-base - coat hanger, flag, guitar, kite, pen, pipe, pliers, sock, spoon, tennis racquet, umbrella, whistle.

Experiment 6: bat, bell, bottle, bowl, chisel, envelope, glass, guitar, lamp, light-bulb, lobster, nut.

\section{References}

[1] Ashbridge E, Perrett DI, Oram MW, Jellema T. Effect of image orientation and size on object recognition: responses of single units in the macaque monkey temporal cortex. Cognitive Neuropsychol 2000;17:13.

[2] Berlin B, Kay P. Basic colour terms: their universality and evolution. Berkeley: University of California Press, 1969.

[3] Biederman I. Recognition-by-components: a theory of human image understanding. Psychol Rev 1987;94:115.

[4] Biederman I, Cooper EE. Evidence for complete translational and reflectional invariance in visual object priming. Perception 1991;20:585.

[5] Bricolo E, Shallice T, Priftis K, Meneghello F. Selective space transformation deficit in a patient with spatial agnosia. Neurocase 2000;6:319.

[6] Cooper ACG, Humphreys GW. Task specific effects of orientation information: Neuropsychological evidence. Neuropsychologia 2000;38:1607-15.
[7] Corballis MC. Recognition of disoriented shapes. Psychol Rev 1988;95:115.

[8] Corballis MC, Beale IL. The psychology of left and right. Hillsdale, NJ: Lawrence Erlbaum, 1976.

[9] Davidoff J, Warrington EK. The bare bones of object recognition: implications from a case of object recognition impairment. Neuropsychologia 1999;37:279.

[10] Davidson HP. A study of the confusing letters b, d, p, q. J Gentic Psychol 1935;47:458.

[11] Deregowski JB, McGeorge P, Wynn V. The role of left-right symmetry in the encodement of spatial orientations. Br J Psychol 2000;91:241.

[12] Farah MJ, Hammond KM. Mental rotation and orientation-invariant object recognition: dissociable processes. Cognition 1988;29:29.

[13] Ferber S, Karnath H-O. Friedrich Best's Case Z with misidentification of object orientation. In: Code C, Wallesch CW, Joanette Y, Lecours AR, editors. Classic cases in neuropsychology, vol. II. East Sussex, Taylor \& Francis, in press.

[14] Funnell MG, Corballis PM, Gazzaniga MS. A deficit in perceptual matching in the left hemisphere of a callosotomy patient. Neuropsychologia 1999;37:1143.

[15] Gold M, Adair JC, Jacobs DH, Heilman KM. Right-left confusion in Gerstmann's syndrome: a model of body centred spatial orientation. Cortex 1995;31:267-83.

[16] Hamm JP, McMullen PA. Effects of orientation on the identification of rotated objects depend on the level of identity. J Exp Psychol Hum Percept Perform 1998;24:413.

[17] Haywood WG. Effects of outline shape in object recognition. J Exp Psychol Hum Perform Percept 1998;24:427.

[18] Hinton GE, Parsons LM. Scene-based and viewer-centered representations for comparing shapes. Cognition 1988;30:1.

[19] Humphreys GW, Romani C, Olson A, Riddoch MJ, Duncan J. Non-spatial extinction following lesions of the parietal lobe in humans. Nature 1994;372:357.

[20] Jolicoeur P. The time to name disoriented natural objects. Mem Cognit 1985;13:289.

[21] Jolicoeur P. Mental rotation and the identification of disoriented objects. Can J Psychol 1989;42:461 published erratum appears in Can J Psychol 1988;43(2): following Table of Contents.

[22] Jolicoeur P. Identification of disoriented objects: a dual systems theory. Mind and Language 1990;5:387.

[23] Jolicoeur P, Milliken B. Identification of disoriented objects: effects of context of prior presentation. J Exp Psychol Learn Mem Cogn 1989;15:200 published erratum appears in J Exp Psychol Learn Mem Cogn 1989;15(4):555.

[24] Karnath HO, Ferber S, Bulthoff HH. Neuronal representation of object orientation. Neuropsychologia 2000;38:1235.

[25] Kosslyn SM, Chabris CF, Marsolek CJ, Koenig O. Categorical versus coordinate spatial relations: computational analyses and computer simulations. J Exp Psychol Hum Percept Perform 1992;18:562.

[26] Langdon DW, Thompson AJ. Relation of impairment to everyday competence in visual disorientation syndrome: evidence from a single case study. Arch Phys Med Rehabil 2000;81:686.

[27] Lawson R. Achieving visual object constancy across plane rotation and depth rotation. Acta Psychol Amst 1999;102:221.

[28] Lawson R, Humphreys GW. View specificity in object processing: evidence from picture matching. J Exp Psychol Hum Percept Perform 1996;22:395.

[29] Lawson R, Humphreys GW. View-specific effects of depth rotation and foreshortening on the initial recognition and priming of familiar objects. Percept Psychophys 1998;60:1052.

[30] Lawson R, Humphreys GW, Jolicoeur P. The combined effects of plane disorientation and foreshortening on picture naming: one manipulation or two? J Exp Psychol Hum Percept Perform 2000;26:568. 
[31] Lawson R, Jolicoeur P. The effect of prior experience on recognition thresholds for plane-disoriented pictures of familiar objects. Mem Cognit 1999;27:751.

[32] Marr D. Vision. San Francisco: WHFreeman, 1982.

[33] Marr D, Nishihara HK. Representation and recognition of the spatial organization of three-dimensional shapes. Proc R Soc Lond B Biol Sci 1978;200:269.

[34] McFie J, Piercey MF, Zangwill OL. Visual spatial agnosia associated with lesions of the right cerebral hemisphere. Brain 1950;73:167.

[35] McMullen PA, Jolicoeur P. The spatial frame of reference in object naming and discrimination of left-right reflections. Mem Cognit 1990;18:99.

[36] Milner AD, Goodale MA. The visual brain in action. Oxford: Oxford University Press, 1995.

[37] Murray JE. Flipping and spinning: spatial transformation procedures in the identification of rotated natural objects. Mem Cognit 1997;25:96.

[38] Ratcliff G. Spatial thought, mental rotation and the right cerebral hemisphere. Neuropsychologia 1979;17:49.

[39] Riddoch MJ, Humphreys GW. Description of a left/right coding deficit in a case of constructional apraxia. Cognitive Neuropsychol 1988;5:289-315.

[40] Rock I. Orientation and form. New York: Academic Press, 1973.

[41] Rollenhagen JE, Olson CR. Mirror-image confusion in single neurons of the macaque inferotemporal cortex. Science 2000;287:1506.

[42] Rudel RG, Teuber HL. Discrimination of direction of line in children. J Comparat Physiological Psychol 1963;56:892.

[43] Rudge P, Warrington EK. Selective impairment of memory and visual perception in splenial tumours. Brain 1991;114:349-60.

[44] Semmes J, Weinstein S, Ghent L, Teuber HL. Correlates of impaired orientation in personal and extrapersonal space. Brain 1963;86:747.

[45] Shepard RN, Metzler J. Mental rotation of three-dimensional objects. Science 1971;171:701.

[46] Snodgrass JG, Vanderwart M. A standardized set of 260 pictures: norms for name agreement, image agreement, familiarity, and visual complexity. J Exp Psychol Hum Learning 1980;6:174.

[47] Solms M, Kaplan SK, Saling M, Miller P. Inverted vision after frontal lobe disease. Cortex 1988;24:499.

[48] Solms M, Turnbull OH, Kaplan-Solms K, Miller P. Rotated drawing: the range of performance, and anatomical correlates, in a series of 16 patients. Brain \& Cognition 1998;38:358-68.

[49] Stankiewicz BJ, Hummel JE, Cooper EE. The role of attention in priming for left-right reflections of object images: evidence for a dual representation of object shape. J Exp Psychol Hum Percept Perform 1998;24:732.

[50] Tarr MJ, Bulthoff HH. Is human object recognition better described by geon structural descriptions or by multiple views? Comment on Biederman and Gerhardstein (1993). J Exp Psychol Hum Percept Perform 1995;21:1494.

[51] Tarr MJ, Pinker S. Mental rotation and orientation-dependence in shape recognition. Cognit Psychol 1989;21:233.

[52] Tarr MJ, Pinker S. When does human object recognition use a viewer-centred reference frame? Psychol Sci 1990;35:567.

[53] Turnbull OH. A double dissociation between knowledge of object identity and object orientation. Neuropsychologia 1997;35:567.

[54] Turnbull OH, Beschin N, Della SS. Agnosia for object orientation: implications for theories of object recognition. Neuropsychologia 1997;35:153.

[55] Turnbull OH, McCarthy RA. Failure to Discriminate between mirror-image objects: a case of viewpoint-independent object recognition? Neurocase 1996;2:63.

[56] Turnbull $\mathrm{OH}, \mathrm{McC}$ arthy RA. When is a view unusual? A single case study of orientation-dependent visual agnosia. Brain Res Bull 1996;40:497.

[57] Vannucci M, Viggiano MP. Category effects on the processing of plane-rotated objects. Perception 2000;29:287.

[58] Warrington EK, Davidoff J. Failure at object identification improves mirror image matching. Neuropsychologia 2000;38:1229.

[59] Warrington EK, James M. Visual object recognition in patients with right-hemisphere lesions: axes or features? Perception 1986; $15: 355$.

[60] Warrington EK, James M. Visual aperceptive agnosia: a clinicanatomical study of three cases. Cortex 1988;24:13.

[61] Warrington EK, James M. The visual object and space perception battery. Bury St. Edmunds: Thames Valley Test Co, 1991.

[62] Warrington EK, Rabin P. Perceptual matching in patients with cerebral lesions. Neuropsychologia 1970;8:475.

[63] Warrington EK, Taylor AM. The contribution of the right parietal lobe to object recognition. Cortex 1973;9:152.

[64] Wechsler D. Wechsler memory scale - revised. San Antonio: Psychological Corporation, 1987.

[65] Zacks J, Mires J, Tversky B, Hazeltine E. Individual differences in mental spatial transformations. Poster presented at Cognitive Neuroscience, San Francisco, 2000.

[66] Zacks J, Rypma B, Gabrieli JD, Tversky B, Glover GH. Imagined transformations of bodies: an fMRI investigation. Neuropsychologia 1999;37:1029. 